\title{
Nerve Tumors: What the MSK Radiologist Should Know
}

\author{
Alberto Stefano Tagliafico, MD ${ }^{1,2}$ Amanda Isaac, FRCR 3,4 \\ Federico Zaottini, MD $^{1}$ Carlo Martinoli, MD ${ }^{1,2}$ \\ 1 Department of Health Sciences (DISSAL), University of Genoa, \\ Genova, Italy \\ 2 IRCCS Ospedale Policlinico San Martino, Genova, Italy \\ 3 Guy's and St Thomas' Hospitals NHS Foundation Trust, London, \\ United Kingdom \\ ${ }^{4}$ King's College London, London, United Kingdom
}

\author{
Address for correspondence Alberto Stefano Tagliafico, MD, \\ Department of Health Sciences (DISSAL), University of Genoa, Via \\ Pastore 1, 16132, Genova, Italy (e-mail: alberto.tagliafico@unige.it).
}

Semin Musculoskelet Radiol 2019;23:76-84.

\author{
Abstract \\ Keywords \\ - peripheral nerves \\ - tumors \\ - sarcoma \\ - ultrasound \\ - magnetic resonance \\ imaging
}

Nerve tumors are rare and heterogeneous soft tissue tumors arising from a peripheral nerve or showing nerve sheath differentiation. In a radiologic setting it is necessary to recognize soft tissue lesions that are of neural origin, their association with a peripheral nerve, and whether they are a true tumor or a so-called pseudotumor such as a neuroma, fibrolipoma, or peripheral nerve sheath ganglion. Ultrasound (US) and magnetic resonance imaging are the best modalities to characterize these lesions. US can be used to guide biopsy in difficult and uncertain cases when the lesion is either indeterminate or possibly malignant. At present, no single imaging feature or reproducible criteria, or a combination, can differentiate reliably between a neurofibroma and a schwannoma or discriminate with certainty between benign and malignant neurogenic tumors. Adequate imaging and consultation with a nerve tumors/sarcoma unit is advised.
Nerve tumors are a subgroup of soft tissue tumors that are rare and heterogeneous. Peripheral nerve sheath tumors (PNSTs) are defined as neoplasms arising from a peripheral nerve or showing nerve sheath differentiation. - Table 1 lists pseudotumors and the World Health Organization classification of the main PNSTs. They account for $\sim 12 \%$ of all benign and $8 \%$ of all malignant soft tissue neoplasms. ${ }^{2,3}$ Benign PNSTs are primarily composed of schwannomas and neurofibromas, each accounting for $5 \%$ of all benign soft tissue tumors, whereas malignant PNSTs account for 5 to $10 \%$ of all soft tissue sarcomas. ${ }^{3}$ It is well known that in patients with a suspicious diagnosis of tumor, an early accurate diagnosis and appropriate treatment are crucial for a good outcome. Therefore, in a radiologic setting it is necessary to recognize soft tissue lesions with a neural origin, their association with a peripheral nerve, and whether they are a true tumor or a pseudotumor such as a neuroma, fibrolipoma, or peripheral nerve sheath ganglion.

Imaging techniques such as ultrasound (US) and magnetic resonance (MRI) imaging are the best modalities to characterize these lesions and have a direct impact on correct management and treatment of patients. In addition, US can be used to guide biopsy in difficult and uncertain cases when the lesion is either indeterminate or there is concern that the lesion is malignant. ${ }^{4}$

Following the guidelines regarding soft tissue tumors in adults approved by the European Society of Musculoskeletal Radiology (ESSR) for peripheral nerve tumors, ${ }^{4}$ biopsy could be avoided in cases of purely benign lesions. The proposed criteria include the following:

- Intraneural ganglia when a well-defined fluid lesion without any solid components and no internal vascularity is visible

- Intraneural lipoma that are homogeneous, well defined, encapsulated, and compressible, with no clinical concern, with documented stability of morphology and size on US (with at least a 6-month follow-up)

- Intraneural vascular malformations with no clinical concern and well-documented morphological stability on US (with at least a 6-month follow-up)

- Morton's neuroma

- Traumatic neuromas

- PNSTs: Only in the case of proven nonpainful neurofibromatosis (NF) when follow-up imaging is used to detect and monitor typical neurofibromas
Issue Theme Bone and Soft Tissue Tumors; Guest Editors, Marc-André Weber, MD, MSc and Radhesh Lalam, MBBS, MRCS, FRCR
Copyright (c) 2019 by Thieme Medical Publishers, Inc., 333 Seventh Avenue, New York, NY 10001, USA. Tel: +1(212) 584-4662.
Dol https://doi.org/ 10.1055/s-0038-1676290. ISSN 1089-7860. 
Table 1 List of pseudotumors and tumors ${ }^{\mathrm{a}}$

\begin{tabular}{|l|}
\hline Pseudotumor \\
\hline Traumatic neuroma \\
\hline Morton's neuroma \\
\hline Intramural ganglia \\
\hline Neural lipomatosis \\
\hline Benign \\
\hline Schwannoma (including variants) \\
\hline Melanotic schwannoma \\
\hline Neurofibroma (including variants) \\
\hline Plexiform neurofibroma \\
\hline Perineurioma \\
\hline Malignant perineurioma \\
\hline Granular cell tumor \\
\hline Dermal nerve sheath myxoma \\
\hline Solitary circumscribed neuroma \\
\hline Ectopic meningioma \\
\hline Nasal glial heterotopia \\
\hline Benign triton tumor \\
\hline Hybrid nerve sheath tumors \\
\hline Malignant \\
\hline Malignant peripheral nerve sheath tumor \\
\hline Epithelioid malignant nerve sheath tumor \\
\hline Malignant triton tumor \\
\hline Malignant granular cell tumor \\
\hline Ectomesenchymoma \\
\hline
\end{tabular}

${ }^{a}$ Slightly modified from the World Health Organization classification of main peripheral nerve sheath tumors (http://whobluebooks.iarc.fr/).

The aim of this practical review is to report the most relevant clinical and radiologic characteristics of common neurogenic tumors or pseudotumors of the peripheral nerves with an emphasis on the role of the musculoskeletal radiologist.

\section{General Recommendations}

Although different centers vary regarding the diagnosis and management of nerve tumors, we recommend following the ESSR guidelines for soft tissue tumors, ${ }^{4}$ and we suggest referral to a specialist peripheral nerve unit or a sarcoma treatment center, specifically in cases of nerve lesions of uncertain origin, to avoid unpleasant surprises when malignant peripheral nerve lesions become evident for the first time during surgery, resulting in intralesional or incomplete resections. ${ }^{4}$

It is important to know and document clinically any relevant trauma, change in size or imaging characteristics, the presence of any underlying genetic disease or oncologic history, the presence of a positive Tinel's sign (denoting the association of the lesion to a nerve), and whether the lesions are multiple or single. This documentation should be reviewed before starting the imaging examination.

\section{Why Image?}

- To assess the type and nature of the lesion

- To look for predictive signs and biomarkers, to assess whether the lesion is benign or malignant

- To assess the disease burden and extent; how many lesions, how big, how far do they extend

- To assess any potential complications: locoregional or associated

- To look for any associations (syndromes, associated malignancies, or lesions in the central nervous system and other organs such as seen in NF1 and NF2)

- To help with posttreatment assessments; whether to evaluate response to therapy or look for recurrence

\section{Technical Requirements}

\section{Conventional Radiography}

Due to inherent low-contrast resolution, radiograph-based techniques are ill suited to evaluate PNSTs.

\section{Ultrasound}

Offering high-resolution imaging, US is quick and easy to perform and can assess vascularity that eliminates having to administer MRI contrast, saving costs and avoiding potential complications. However, it is sometimes limited in assessing the extent of the lesion when deep or when the lesion shows low contrast to surrounding structures. It is also operator dependent and therefore less reliable in the assessment of interval change. US equipment should incorporate at least $12-\mathrm{MHz}$ probes, images should be stored in a picture archival communication system, and a written report must be available for each patient. Peripheral nerve US is sometimes performed in clinics in nonradiologic settings, as part of the clinical examination, to evaluate the possible presence of a soft tissue mass. However, to confirm it is of neurogenic origin, a board-certified radiologist with proven experience in sonographic soft tissue lesion assessment should perform the US. Indeed, it was estimated that at least $5.4 \%$ of peripheral nerve US studies led to additional clinical or imaging assessment ${ }^{5}$ due to the presence of nonneural structures worthy of subspecialized radiologic assessment.

\section{MRI Imaging}

MRI is the gold standard for superficial and deep lesions. Utilizing MRI it is possible to characterize the lesions: size, intratumoral lobulations, morphology, margins (well or ill defined) and detect perilesional edema, cystic changes (necrosis), interval change in size, vascularity, and effects on surrounding structures. The benefits of MR imaging include:

- It is less operator dependent.

- It can assess deep structures more confidently.

- It can cover a larger body part (or in whole-body MRI, the whole body).

- It can assess for skip lesions that often occur along the nerve.

The potential downside of MRI is that is relatively more time consuming and less available than US. 
US and MRI are commonly used alone or in combination to study peripheral nerves. In general, US is well suited for very small and superficial structures, whereas MRI is good for deeper lesion such as those located deep in the brachial plexus, lumbar plexus, and in patients with a high body mass index. US is often the imaging modality initially used to study peripheral nerves in centers where both US and MRI are available.

\section{Positron Emission Tomography/Computed Tomography Imaging}

Positron emission tomography/computed tomography (PET/ $\mathrm{CT}$ ) is a valuable functional imaging diagnostic tool and can help differentiate reliably between benign and malignant lesions. However, PET/CT remains relatively expensive and is associated with a high radiation dose, which is important in this particular cohort of young patients who often require regular imaging. We therefore have to consider carefully how and when we image with PET/CT.

On PET/CT, high uptake (maximum standard uptake value maximum [SUVmax] $>3$ ) and increased SUV on delayed imaging are indicators for malignancy. Delayed SUVmax is a useful parameter to assess because in benign PNST, the SUVmax tends to drop over time, whereas SUVmax increases in malignant PNST SUV.

To date, there are no established values for the optimal SUVmax cut-offs in the published literature. The use of an SUVmax value of 3.5 as a cut-off was adopted by some trials, ${ }^{6}$ suggested as the optimum SUVmax cut-off to maximize sensitivity and specificity. The range varies; however, there remains debate about those cases when the SUVmax value lies between 3.0 and 4.0 .

\section{Written Report}

As for other soft tissue masses, in case of nerve tumors the report should include the relation of the lesion to the surrounding fascia (superficial, deep), the exact anatomical location and the nerve of origin, the relationship with the main anatomical vascular and tendinous structures to the lesion, and its size in three dimensions including the longitudinal involvement of the nerve of origin. Finally, if there is any suspicious of malignancy, MRI should be performed to comment on the extent of locoregional disease. - Table 2 presents the criteria used to suspect the presence of a neurogenic tumor.

\section{Purely Benign Lesions}

Rare benign lesions affecting peripheral nerves are perineurioma (differentiated perineurial cells inside or outside a nerve), intraneural lipoma and/or lipofibromatous hamartoma (fibrofatty tumor characterized by the proliferation of mature adipocytes within the epineurium and the perineurium of the peripheral nerves), ${ }^{7-9}$ and intraneural vascular malformations. ${ }^{10,11}$ These are called purely benign because they have no predilection for malignant transformation. Detailed description of these very rare entities are beyond the scope of this practical review.

\section{Morton's Neuroma}

Morton's neuroma is a common nonneoplastic fusiform enlargement of the plantar digital nerve due to perineural fibrosis, local vascular proliferation, edema of the endoneurium, and axonal degeneration. ${ }^{12}$ The plantar digital nerve of the third intermetatarsal space is most commonly affected, followed by the plantar digital nerve of the second intermetatarsal space. ${ }^{12}$ The presence of Morton's neuroma is one of several causes of metatarsalgia; therefore, diagnostic imaging is often requested when the clinical examination is equivocal. US and MRI are both sensitive and reliable in a patient with metatarsalgia to diagnose Morton's neuroma. ${ }^{13}$ According to a 2015 metaanalysis, MRI is not superior to US for the diagnosis of Morton neuroma. ${ }^{13}$ Morton's neuroma appears as a hypoechoic intermetatarsal mass. Identification of the presumed plantar digital nerve in continuity with the mass improves diagnostic confidence, especially with a dorsal approach, but this is not specific. The Mulder's clinical test, when performed during US assessment of the forefoot, may increase diagnostic confidence. Size and symptoms are not absolutely related, although most symptomatic neuromas are $>5 \mathrm{~mm}$ in diameter. ${ }^{12}$ Using MRI, Morton's neuroma is better demonstrated on coronal or axial small field-of-view T1-weighted images. Established criteria for diagnosis include the following:

- The lesion is centered within the neurovascular bundles in the intermetatarsal space and on the plantar side of the transverse intermetatarsal ligament.

- The signal intensity of the lesion mirrors that of skeletal muscle on T1-weighted images and shows lower signal than that of fat on T2-weighted images (likely reflecting the lesion's high collagen content and/or fibrosis). ${ }^{14}$

- Intermetatarsal bursal fluid proximal and dorsal to Morton's neuroma may be a frequent associated finding. A large amount of intermetatarsal bursal fluid $(>3 \mathrm{~mm}$ in transverse diameter) or fluid in the fourth intermetatarsal should suggest a diagnosis of a Morton's neuroma. ${ }^{14}$

\section{Traumatic Neuromas}

Traumatic neuromas are nonneoplastic proliferation of the proximal end of a partially or completely transected nerve. In these cases, the clinical history is often straightforward, even when a soft tissue mass at a focal pressure is evident. It is often possible to reproduce pain with palpation with or without the Tinel's sign. Traumatic neuromas have a "bulbous-end" morphology, lie peripherally, and appear to be in continuity with a normal nerve proximally. ${ }^{12}$ Histologically, traumatic neuromas correspond to nonneoplastic, nonencapsulated tangled masses of axons, Schwann cells, endoneurial cells, and perineurial cells in a dense collagenous matrix with surrounding fibroblasts. Disorganization of the neurogenic tissue (caused by multidirectional proliferation of cells in an abortive attempt to repair the injured nerve) allows traumatic neuromas to be distinguished from neurofibroma. ${ }^{12}$ US and MRI show a fusiform mass with normal appearances of an entering and exiting nerve, or only an entering nerve terminating in a bulbous shape. ${ }^{12,15}$ On US the neuroma is homogeneously hypoechoic 
Table 2 Imaging criteria for suspicion of a neurogenic tumor

\begin{tabular}{|l|l|l|}
\hline Imaging feature & Description & Note \\
\hline $\begin{array}{l}\text { Growth into a } \\
\text { major nerve }\end{array}$ & Good for diagnosis & \\
\hline $\begin{array}{l}\text { Entering and } \\
\text { exiting nerve }\end{array}$ & $\begin{array}{l}\text { Most reliable feature to } \\
\text { suggest a neurogenic origin }\end{array}$ & Impossible in superficial or in small lesions \\
\hline Fusiform shape & Related to the intraneural growth & Not present in soft tissue sarcomas \\
\hline Split-fat sign & Peripheral rim of fat visualized on MRI, US, or CT & More common in PNSTs and large nerves \\
\hline Target sign & Visible on US and MRI fluid-sensitive sequences & $\begin{array}{l}\text { Fibrous tissue centrally and myxoid } \\
\text { tissue peripherally at histology }\end{array}$ \\
\hline $\begin{array}{l}\text { Atrophy of the } \\
\text { innervated muscles }\end{array}$ & $\begin{array}{l}\text { Possible but infrequent and denotes } \\
\text { associated neural compromise; } \\
\text { EMGs are advised to evaluate neural function }\end{array}$ & Not reliable for diagnosis \\
\hline
\end{tabular}

Abbreviations: CT, computed tomography; EMG, electromyelogram; MRI, magnetic resonance imaging; PNST, peripheral nerve sheath tumor; US, ultrasound.

with no appreciable degenerative or necrotic pseudocystic foci present. On MRI, traumatic neuromas have intermediate signal intensity (similar to that of muscle) onT1-weighted MR images and intermediate to high signal intensity on T2-weighted images. $^{12,15}$

\section{Schwannoma and Neurofibroma (Benign PNSTs)}

Both schwannomas and neurofibromata are made of cellular elements closely related to normal Schwann cells, although some pathologic features usually allow distinction.

Schwannoma (neurilemoma) represents $\sim 5 \%$ of all benign soft tissue neoplasms and commonly involves the spinal and sympathetic nerve roots of the head and neck, as well as nerves in the flexor surfaces of the upper and lower extremities (particularly ulnar and peroneal nerves). ${ }^{12}$

Schwannomas are usually solitary lesions with benign features. Only $5 \%$ of multiple plexiform schwannomas can be associated with NF2. ${ }^{16}$

Both the schwannoma and the nerve of its origin grow within the epineurium. On histology Antoni A and Antoni B regions are typical of schwannomas, as well as S-100 protein positivity. Large schwannomas (ancient schwannomas) can undergo degenerative changes with cyst formation, calcification, hemorrhage, and even focal fibrosis. ${ }^{15}$ During surgery, it is possible to separate the neoplasm from the native nerve and to preserve its function (-Figs. 1 and $\mathbf{2}$ ). Recurrence is unusual. Malignant transformation is exceedingly rare. $^{12}$ Schwannoma is distinctly eccentric and therefore separable from normal nerve, whereas neurofibroma is not (-Fig. 1).

Neurofibromata, in contrast, are typically located centrally in respect to nerve of origin and exhibit a target sign on T2weighted images, with small cystic cavitation, foci of necrosis, or calcifications when compared with schwannomas. ${ }^{12,16}$

Often the differentiation between a schwannoma and neurofibroma is not easy, and to date, we are not aware of a prospective study reporting diagnostic accuracy or algorithms to differentiate the two lesions reliably.

Although most PNSTs are sporadic, multiple schwannomas are seen in most patients with NF2. Similarly, most neurofibromata are sporadic lesions, but multiple, plexiform, and large neurofibromata are almost always associated with NF1. Some, if not all, ganglioneuromas may arise as a result of maturation of neuroblastomas or other neuroblastic tumors.
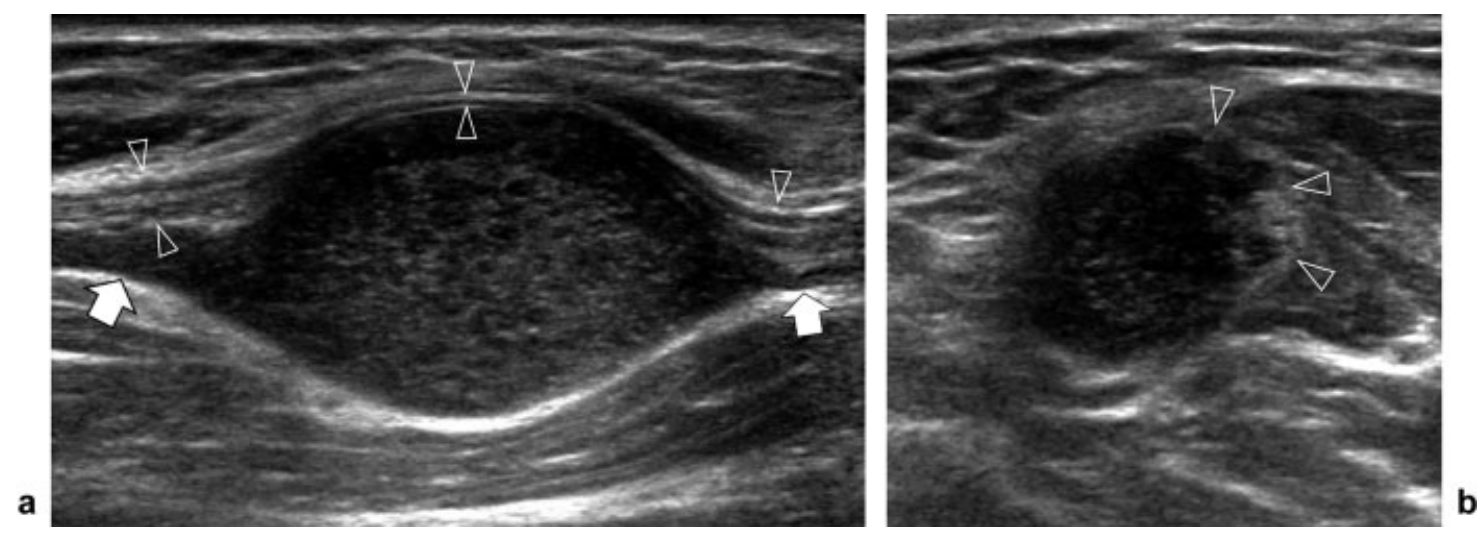

Fig. 1 Schwannoma. (a) Long-axis and (b) short-axis 17-5 MHz US images over the ulnar nerve in the arm demonstrate a hypoechoic oval mass in continuity with the nerve at its proximal and distal poles. The tumor is connected with a swollen fascicle (arrows), whereas the other fascicles (arrowheads) remain unaffected and are displaced at the periphery. 

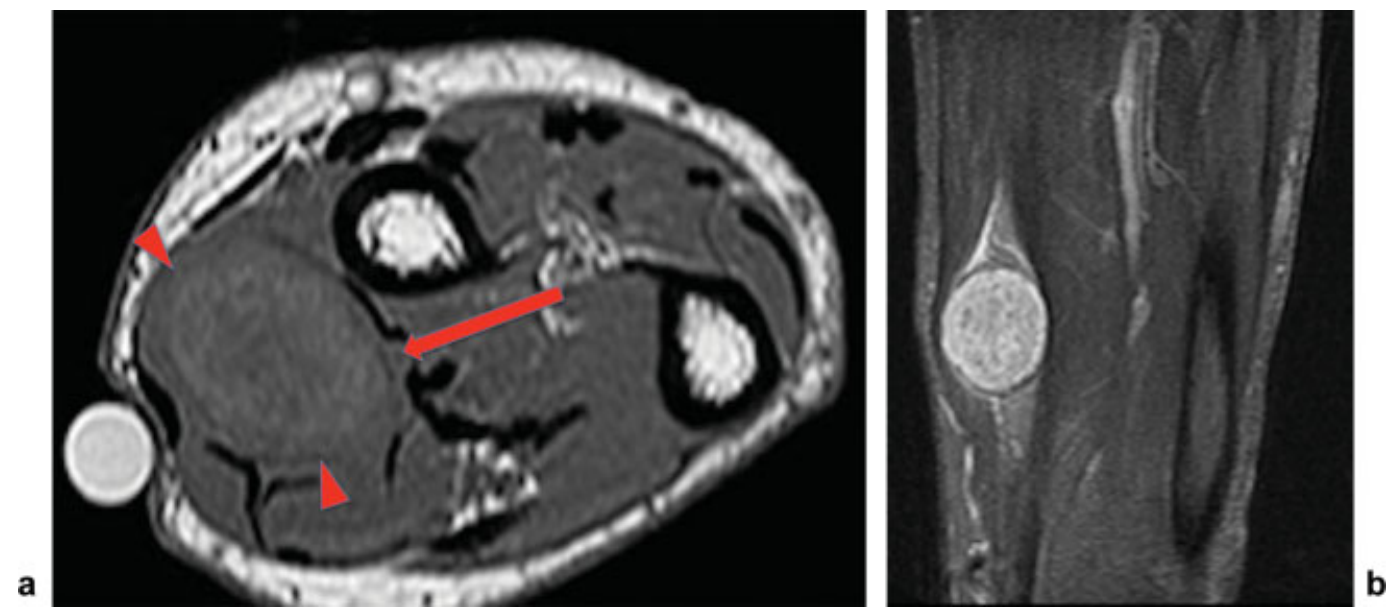

Fig. 2 Schwannoma. (a) T1 axial image on the left shows a well-defined mildly heterogeneous soft tissue mass that is isointense to slightly hyperintense to adjacent muscle. The mass is in the expected location of the median nerve. Red arrowheads indicate the tumor, red arrow indicates the expected location of the median nerve. (b) Coronal short tau inversion recovery imaging demonstrates a fusiform soft tissue mass associated with the neurovascular bundle that is diffusely hyperintense. The mass is surrounded by a thin low signal intensity margin with a characteristic "fat splitting" sign.

Ganglioneuromas can occasionally be seen in lymph nodes of patients with a remote history of neuroblastoma.

Typical MRI signs of benign PNSTs are presented in - Fig. 3. -Figs. 4, 5, 6, 7, 8 show other examples of benign PNSTs.

\section{Malignant PNSTs}

Malignant PNSTs can be either solitary or associated with NF1 (in 25-70\% of cases). They can be considered high-grade sarcomas. ${ }^{16}$ Major nerve trunks are commonly involved by malignant PNSTs. The clinical symptoms are generally worse than in patients with benign PNSTs. Notably, the onset of symptoms is usually rapid, and pain at rest is typical in malignant PNSTs but not in benign PNSTs.

As with other secondary tumors, malignant PNSTs can follow radiation therapy (often recorded after several years from exposure).
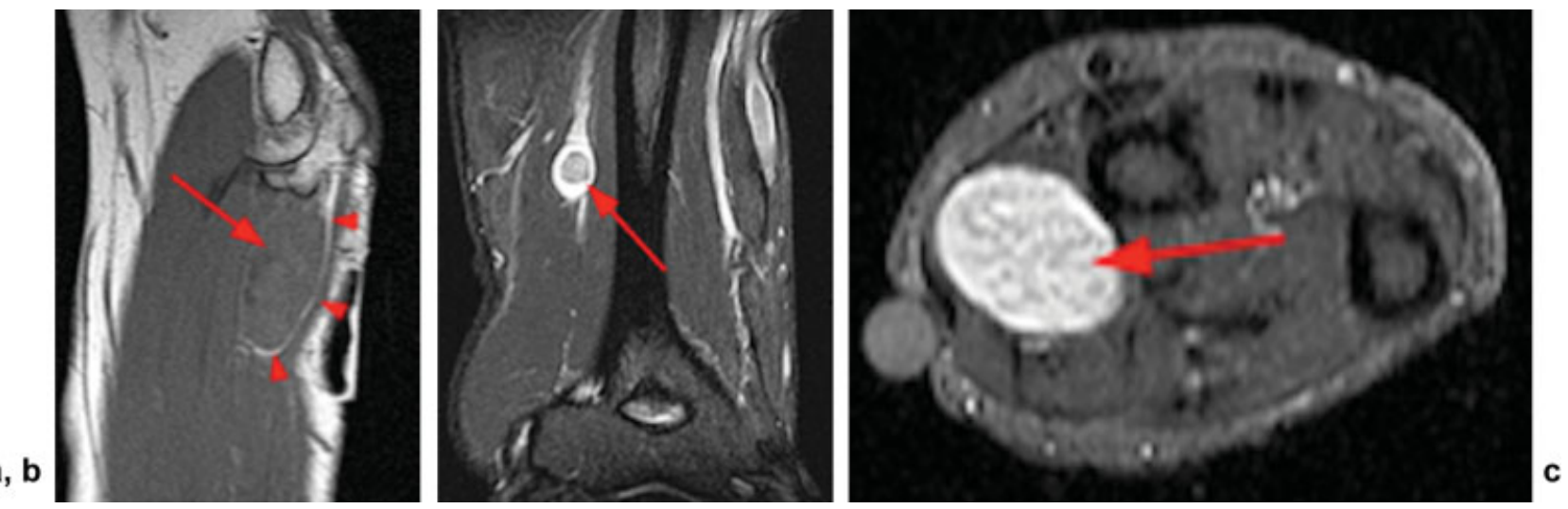

Fig. 3 Classic MRI signs on MR Imaging A, B, and C of benign peripheral nerve sheath tumors (PNSTs). (a) Split fat sign. A sagittal T1-weighted image obtained through a histologically proven schwannoma of the ulnar nerve in a 59 -year-old man. The fusiform mass is surrounded by a thin margin of fat. This appearance results as the mass within the neurovascular bundle enlarges and displaces the adjacent intramuscular fat. (b) Target sign. Coronal short tau inversion recovery (STIR) image through a schwannoma of the radial nerve. The lesion is of high signal intensity peripherally and low signal centrally. It is caused by a zonal pattern of architecture of the lesion, with more myxoid material peripherally and more fibrous tissue centrally. This can be seen in either a benign PNST or a malignant PNST and can cause errors in diagnosis. (c) Fascicular sign. Axial STIR image in the same patient with multiple ring-like structures are seen throughout the lesion. Schwannoma seems to be more commonly associated with a fascicular appearance than neurofibroma. Red arrowheads and arrows indicate the tumor. 
a
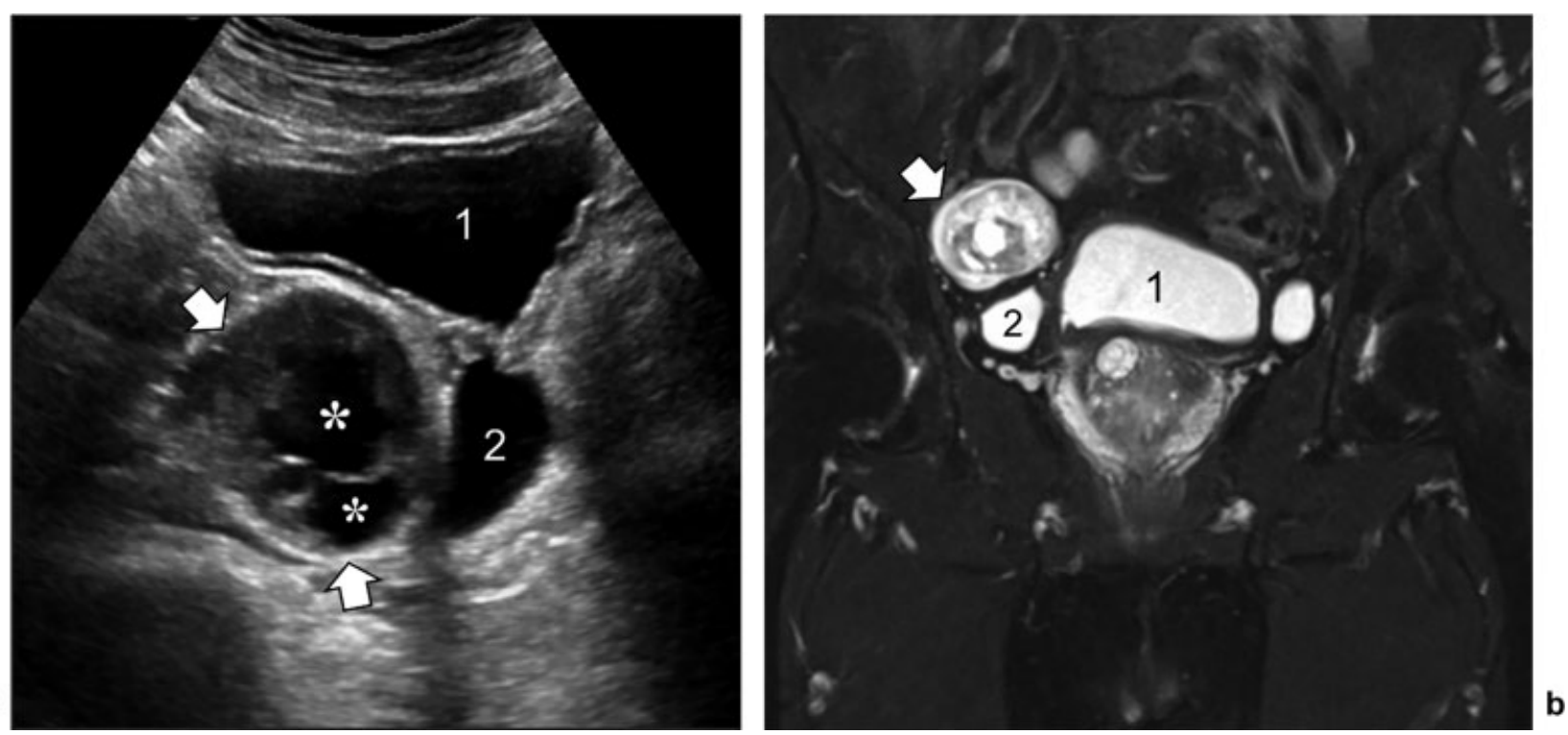

Fig. 4 Cystic schwannoma. (a) Transverse 5-1 MHz US image of the pelvis (b) with coronal fat-suppressed T2-weighted MR imaging correlation show a globoid mixed mass (arrows) consisting of solid hypoechoic and fluid (asterisks) components along the intrapelvic course of the right obturator nerve. The tumor is located on the right of the urinary bladder (1) and in proximity to a bladder diverticulum (2).

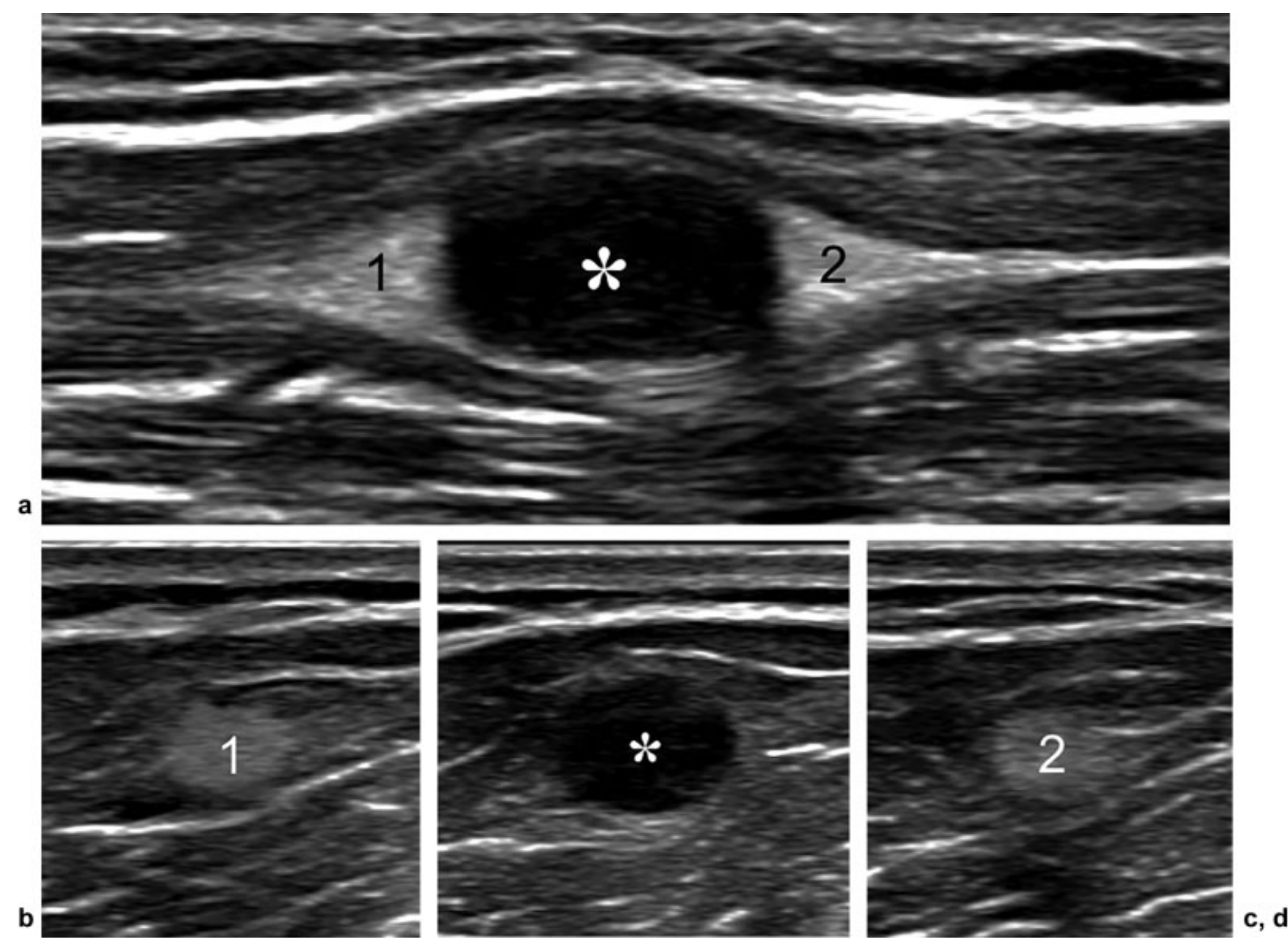

Fig. 5 Fat-split sign. (a) Long-axis and (b-d) corresponding short-axis 22-2MHz ultrasound images of a small intramuscular schwannoma (asterisk) reveal a tapered cap of hyperechoic fat adjacent to the proximal (1) and distal (2) ends of the tumor.

imaging, minimum apparent diffusion coefficient values usually are $>1.0$ to $1.110^{-3}$ in benign lesions and $<1.0-1.210^{-3}$ in malignant lesions. On spectroscopy, the trimethylamine fraction is usually $<50 \%$ in benign lesions and $>50 \%$ in malignant lesions. ${ }^{17}$ NF could be associated with several complications and syndromes ${ }^{18}$ reported here in order of frequency: café-au- 


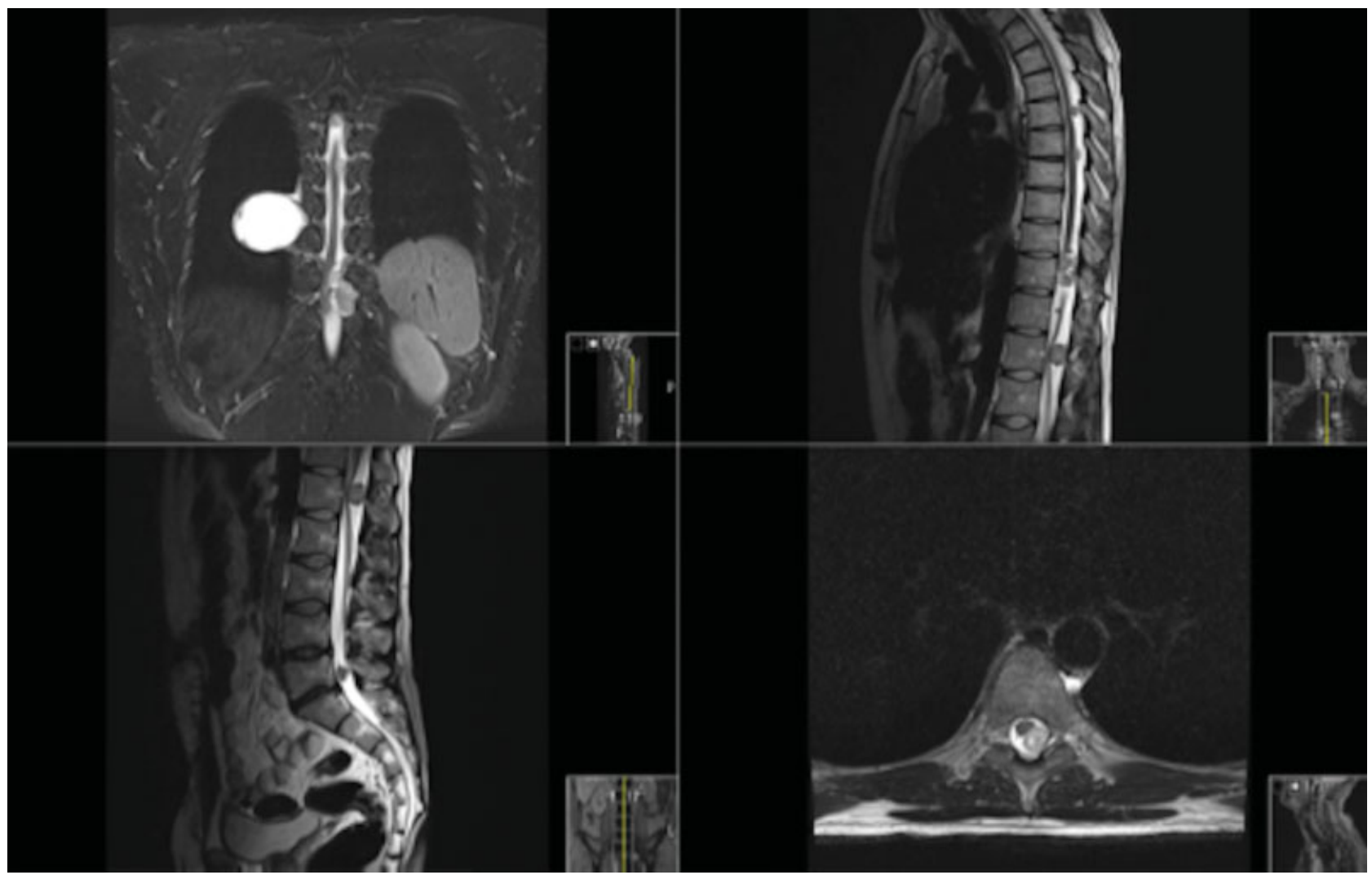

Fig. 6 Multiplanar MRI imaging in a patient with schwannomatosis, with multiple intrathoracic and intradural extramedullary lesions. MRI is crucial for evaluation of these deep lesions at the time of the initial diagnosis as well as for follow-up.

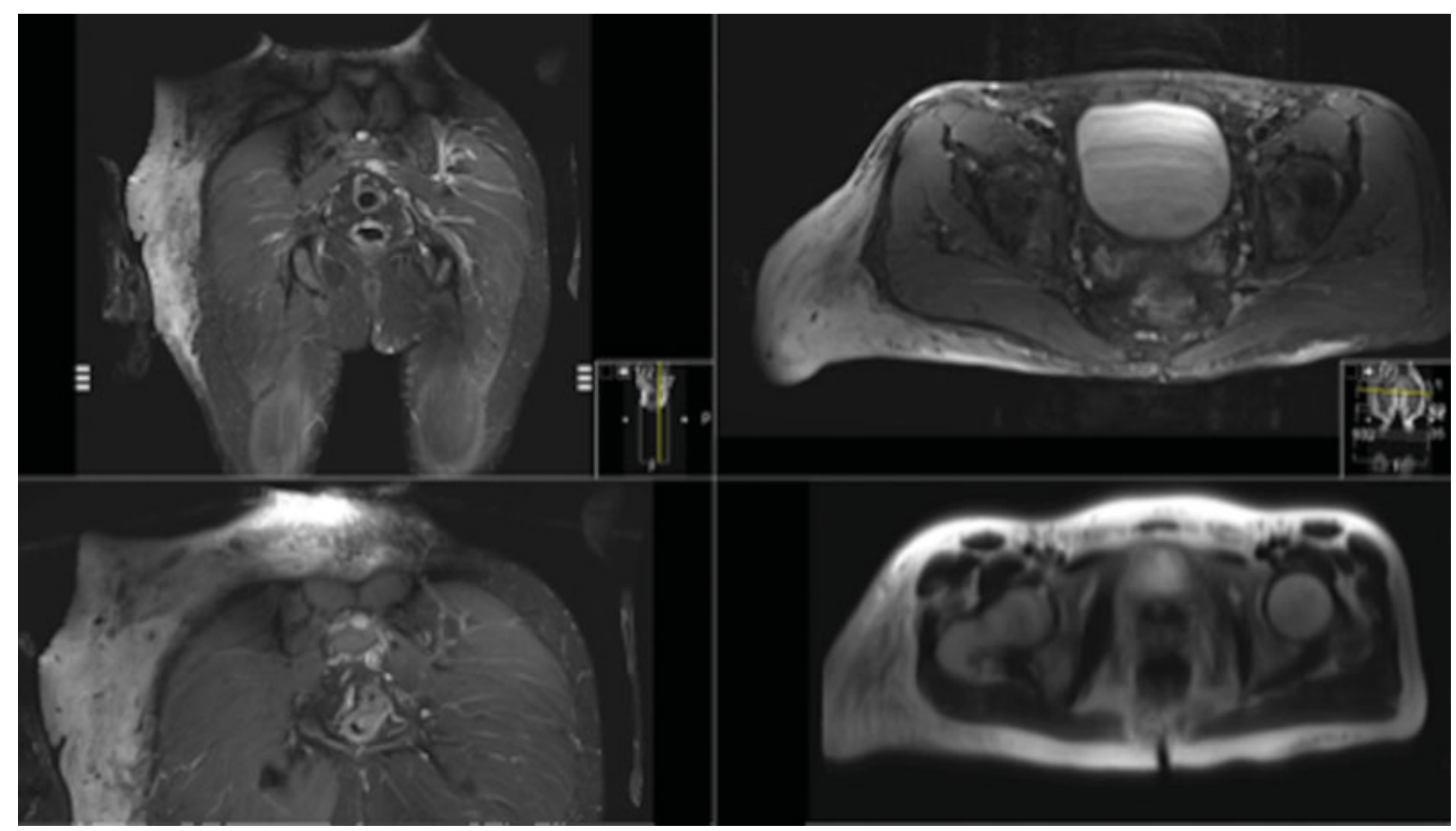

Fig. 7 Multiplanar MRI imaging of a cutaneous plexiform neurofibroma arising in a neurofibromatosis 1 patient. Note that the lesion is limited to skin and subcutaneous tissues with no extension into the underlying muscles.

lait patches (99\%), skin-fold freckling (85\%), Lisch nodules (90$95 \%)$, cutaneous neurofibromas (99\%), plexiform neurofibromas (30-50\%), disfiguring facial plexiform neurofibromas (3-5\%), malignant PNST (2-5\%), scoliosis (10\%), scoliosis requiring surgery (5\%), pseudarthrosis of tibia (2\%), renal artery stenosis (2\%), phaeochromocytoma (2\%), severe cognitive impairment 


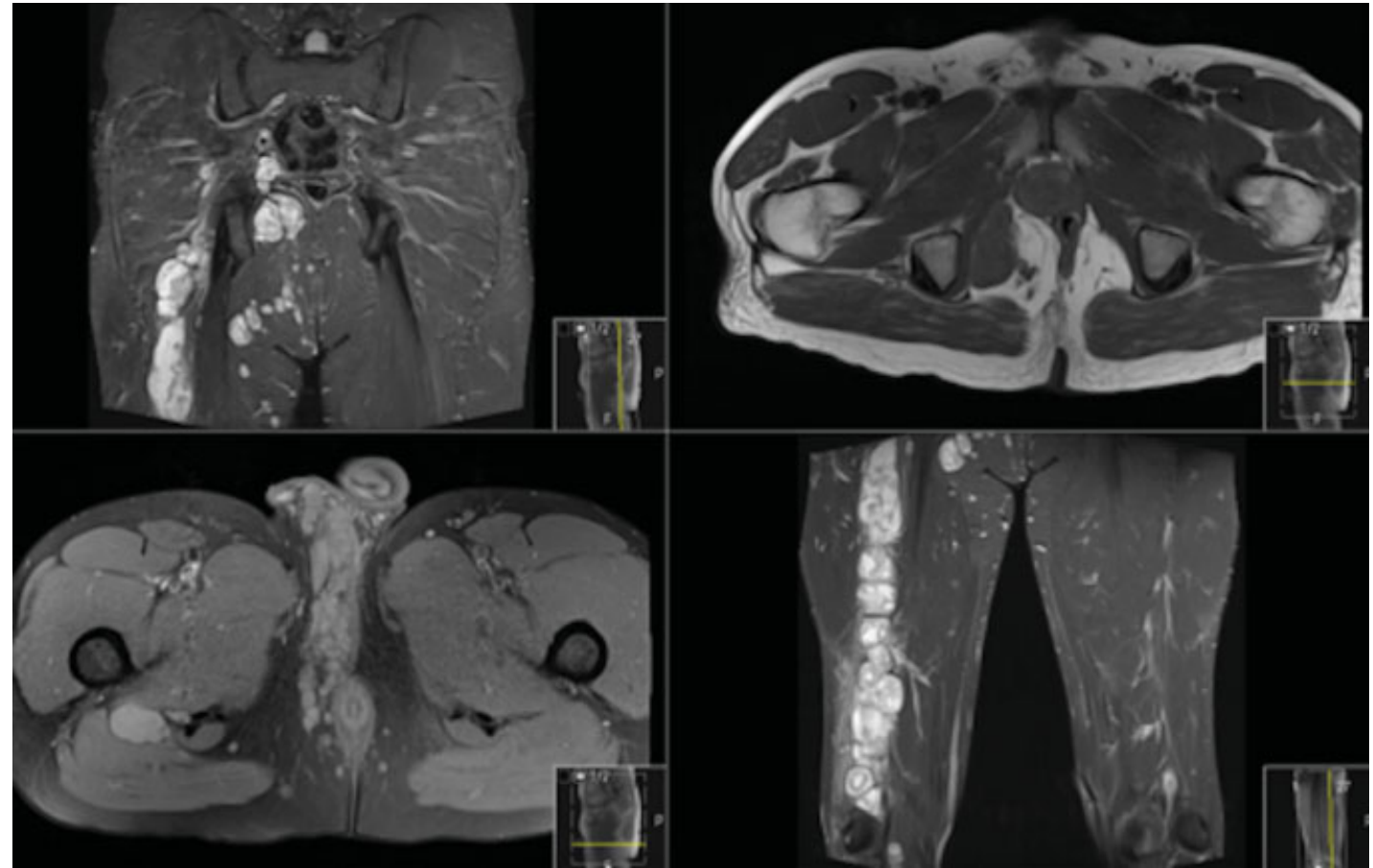

Fig. 8 Another case of a complex lumbosacral plexiform neurofibroma arising in a neurofibromatosis 1 patient and extending into the scrotum and pudendal nerve.
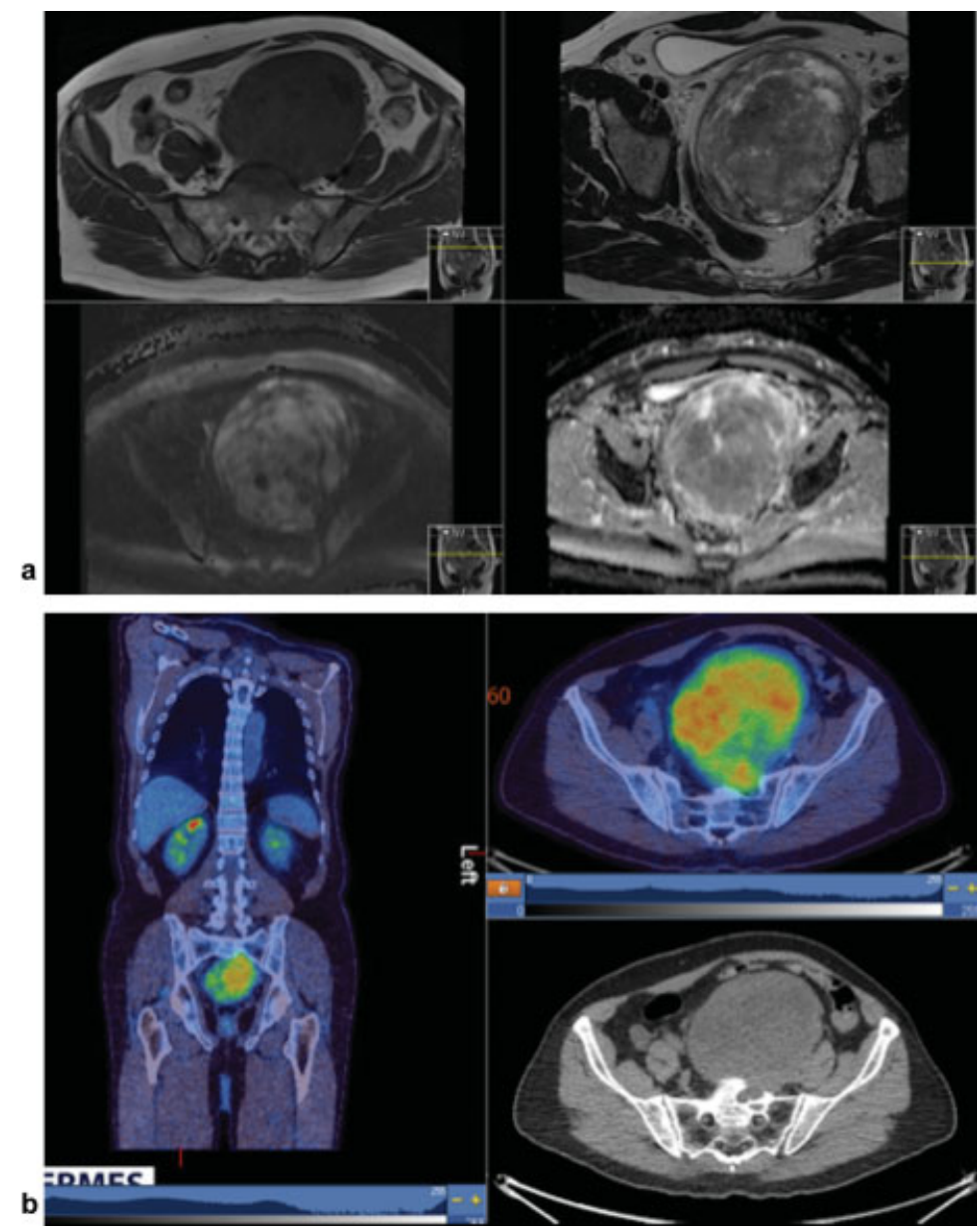

Fig. 9 A presacral mass that was invading the sacrum with marrow edema, perilesional edema, a heterogenous signal (a), and a standard uptake value of 8 (b). On excision, it was confirmed histologically to be a malignant peripheral nerve sheath tumor. 
(4-8\%), learning problems (30-60\%), epilepsy (6-7\%), optic pathway glioma $(15 \%)$, cerebral gliomas $(2-3 \%)$, sphenoid wing dysplasia $(<1 \%)$, and aqueduct stenosis $(<1 \%)$.

\section{Future Directions}

Differentiation between large benign PNSTs and malignant PNSTs remains a diagnostic challenge. Thus future research could focus efforts in the differentiation of these entities. Evolving technologies include the use of quantitative imaging including radiomics, enhancement patterns, and spectroscopy of nerve tumors. In addition, incorporating whole-body functional imaging using whole-body MRI may improve the diagnosis and follow-up of patients but requires dedicated training and precise protocols to reduce the risk of missing small lesions on those fairly large field-of-view sequences.

Adopting a multidisciplinary approach improves patient outcomes and reduces potential morbidity and mortality relating to the disease process. We would encourage close collaboration between clinicians and radiologists to facilitate a uniform approach to the diagnosis and management of nerve tumors and effectively manage any complications. Furthermore, the formation of a standardized database will facilitate the evaluation of care and long-term response to treatment for these complex lesions. Ideally, multidisciplinary nerve tumor clinics could include a lead clinician, named consultants who are experts in their field, and a specialist nurse. The disciplines of neurology, pediatrics, genetics, ophthalmology, neurosurgery, plastic surgery, orthopaedics, soft tissue tumor surgery, psychiatry, dermatology, radiology, and pathology should be represented.

Reliable clinical and radiologic assessment will be helpful in determining the value of potential therapies. A dedicated specialized service also provides a forum for clinical audit and academic interaction through multidisciplinary meetings and video conferencing.

Dedicated specialist clinics help diagnose PNST in difficult cases, depict malignant transformation, monitor and manage complex disease, as well as educate and support the patient and family.

\section{Summary}

Peripheral nerve tumors are rare, but with a combination of different imaging techniques, mainly US and MRI, it is possible to characterize neurogenic tumors confidently, with a great impact on patient management. At present, there is no single imaging feature or reproducible criteria of a combination of features for reliable differentiation between neurofibroma and schwannoma or for confident discrimination between benign and malignant neurogenic tumors. Adequate imaging and discussion with a nerve tumor/sarcoma unit is therefore warranted. A multidisciplinary approach for patient care is advised when possible.

\section{References}

1 Fletcher CDM, Bridge JA, Hogendoorn P, Mertens F. WHO Classification of Tumours of Soft Tissue and Bone. 4th ed. Lyon, France: IARC Press; 2013

2 National Cancer Institute. SEER Stat Fact Sheets: Soft tissue including heart cancer. Available at: http://seer.cancer.gov/statfacts//. Accessed July 8, 2018

3 Kerezoudis P, Bydon M, Spinner RJ. Peripheral nerve sheath tumors: the "orphan disease" of national databases. World Neurosurg 2017;103:948-949

4 Noebauer-Huhmann IM, Weber MA, Lalam RK, et al. Soft tissue tumors in adults: ESSR-approved guidelines for diagnostic imaging. Semin Musculoskelet Radiol 2015;19(05):e1

5 Bignotti B, Zaottini F, Airaldi S, Martinoli C, Tagliafico A. Extraneural findings during peripheral nerve ultrasound: prevalence and further assessment. Muscle Nerve 2018;57(01): 65-69

6 Warbey VS, Ferner RE, Dunn JT, Calonje E, O’Doherty MJ. [18F]FDG $\mathrm{PET} / \mathrm{CT}$ in the diagnosis of malignant peripheral nerve sheath tumours in neurofibromatosis type-1. Eur J Nucl Med Mol Imaging 2009;36(05):751-757

7 Afshar A, Assadzadeh O, Mohammadi A. Ultrasonographic diagnosis of lipofibromatous hamartoma of the median nerve. Iran J Radiol 2015;12(01):e11270

8 Johnson RJ, Bonfiglio M. Lipofibromatous hamartoma of the median nerve. J Bone Joint Surg Am 1969;51(05):984-990

9 Shekhani HN, Hanna T, Johnson JO. Lipofibromatous hamartoma of the median nerve: a case report. J Radiol Case Rep 2016;10(11): $1-7$

10 Rusu GM, Ciuce C, Fodor L, Manole S, Dudea SM. Ultrasonographic and imaging appearance of peripheral intraneural vascular anomalies: report of two cases and review of the literature. Med Ultrason 2018;20(02):237-246

11 Prasad NK, Chawla A, Lalezari S, et al. A radiological classification system for intraneural vascular anomalies: assessment of potential for resection with high-resolution MRI. Acta Neurochir (Wien) 2016;158(02):329-334; discussion 334

12 Abreu E, Aubert S, Wavreille G, Gheno R, Canella C, Cotten A. Peripheral tumor and tumor-like neurogenic lesions. Eur J Radiol 2013;82(01):38-50

13 Bignotti B, Signori A, Sormani MP, Molfetta L, Martinoli C, Tagliafico A. Ultrasound versus magnetic resonance imaging for Morton neuroma: systematic review and meta-analysis. Eur Radiol 2015; 25(08):2254-2262

14 Zanetti M, Ledermann T, Zollinger H, Hodler J. Efficacy of MR imaging in patients suspected of having Morton's neuroma. AJR Am J Roentgenol 1997;168(02):529-532

15 Tagliafico A, Altafini L, Garello I, Marchetti A, Gennaro S, Martinoli C. Traumatic neuropathies: spectrum of imaging findings and postoperative assessment. Semin Musculoskelet Radiol 2010;14 (05):512-522

16 Murphey MD, Smith WS, Smith SE, Kransdorf MJ, Temple HT From the archives of the AFIP. Imaging of musculoskeletal neurogenic tumors: radiologic-pathologic correlation. Radiographics 1999;19(05):1253-1280

17 Soldatos T, Fisher S, Karri S, Ramzi A, Sharma R, Chhabra A. Advanced MR imaging of peripheral nerve sheath tumors including diffusion imaging. Semin Musculoskelet Radiol 2015;19(02): 179-190

18 Ferner RE, Huson SM, Thomas N, et al. Guidelines for the diagnosis and management of individuals with neurofibromatosis 1. J Med Genet 2007;44(02):81-88 